\title{
Study on Correlation Between Coating Rate and Hot Water Soluble Substances of Reconstituted Tobacco
}

\author{
Dandan $\mathrm{Su}^{1}$, Guiyuan Zhou ${ }^{1}$, Wenjun Zhang ${ }^{1,}$, Dejun $\mathrm{Wu}^{2}$, Xiaosheng Wang ${ }^{1}$, Hua Chen ${ }^{1}$, \\ Mingwen Wei ${ }^{1}$, Tao Feng ${ }^{1}$ \\ ${ }^{1}$ China Tobacco Schweitzer (Yunnan) Reconstituted Tobacco Co. Ltd, Yuxi, China \\ ${ }^{2}$ Yantai Zhonghai Diaotai Biotechnology Development Co. Ltd, Yantai, China
}

\section{Email address:}

724093057@qq.com (Dandan Su), zhouguiyuan@cts-yn.com (Guiyuan Zhou), zhangwenjun@cts-yn.com (Wenjun Zhang)

${ }^{*}$ Corresponding author

\section{To cite this article:}

Dandan Su, Guiyuan Zhou, Wenjun Zhang, Dejun Wu, Xiaosheng Wang, Hua Chen, Mingwen Wei, Tao Feng. Study on Correlation Between Coating Rate and Hot Water Soluble Substances of Reconstituted Tobacco. American Journal of Agriculture and Forestry.

Vol. 6, No. 4, 2018, pp. 65-70. doi: 10.11648/j.ajaf.20180604.11

Received: June 10, 2018; Accepted: June 26, 2018; Published: July 18, 2018

\begin{abstract}
The corresponding relationship between the coating rate and hot water soluble of paper-making reconstituted tobacco was studied in this paper which provided references for the research and application on the off-line detecting of coating rate. Through the accurate quantitative coating experiment, the hot water soluble of reconstituted tobacco by paper-making was determined through soxhlet extraction and coffee machine washing in this paper. And the corresponding relationship between the coating rate and hot water soluble determined by these two methods was also compared in order to make sure which method was more suitable. The results showed that the hot water soluble value was significantly positively correlated with coating rate $(\mathrm{P}<0.01)$, if the coating rate distribute between $38 \%$ and $46 \%$. The linear equation obtained $\mathrm{y}=1.007 \mathrm{x}+1.387, \mathrm{R}^{2}=0.974$ and $y=0.932 x+3.181, R^{2}=0.985$, respectively. The hot water soluble value determined by these two methods were different from each other. And the values determined by soxhlet extraction were mostly lower than the values detected by coffee machine washing; The coating rate derived from hot water soluble values determined by coffee machine washing was more closer to the actual values. It is feasible to derive the coating rate from hot water soluble values, and the method of coffee machine washing is more suitable for practical production testing, which has the advantage of short testing time and simple operation.
\end{abstract}

Keywords: Papermaking Reconstituted Tobacco, Coating Rate, Hot Water Soluble, Correlation

\section{Introduction}

Paper-making reconstituted tobacco (PRT) is a kind of product which can reduce the tar release amount effectively, its quality affects the quality of cigarette products directly, and the coating process is key to the whole produce process [1-2], well-controlled coating rate has great influence on reducing the fluctuation of product physical and chemical indicators and keeping the stability of product quality [3-4, 13-14]. As there is no uniform testing method and error requirement for coating rate in China, various industries including coating industry have different calculation methods for coating rate $[5-6,15-16]$, and can only be calculated online while producing [7-8]; For cigarette manufacturing enterprises, the reconstituted tobacco products they receive are mostly coated products in pieces of different sizes, and the coating rate cannot be detected by taking round sheet of $100 \mathrm{~cm}^{2}$ on-line. Therefore, developing the testing method of coating rate, especially the off-line testing method, is of great practical significance for cigarette manufacturing enterprises to control the production process and stabilize product quality.

At present, there are few domestic and international reports on the testing method of coating rate of paper-making reconstituted tobacco, especially the development of off-line testing method. Qiu Ye etc. [10] extracted the soluble substance in finished products and base sheet of paper-making reconstituted tobacco respectively through the method of soxhlet extraction, and the extraction yield of the finished product and the base sheet were calculated respectively, and the coating rate was calculated through formula. Hongta 
tobacco (group) co., LTD published a testing method of hot water soluble of paper-making reconstituted tobacco in 2013, paper-making reconstituted tobacco was grinded into particles with certain sizes, and heating refluxed in water for a certain period, after being cooled and filtered the content of hot water soluble was obtained by calculating the difference between the initial sample quality and the residual quality after filtration. DAI Li-jun [11] used the principle of same materials has similar limit extraction yield, modified the calculation formula of extraction yield of hot water soluble, the modified formula could be used to calculate the extration yield of hot water soluble for online materials of reconstituted tobacco. LTR Industry Co. Ltd. in France, as an international manufacturing company who owns the most advanced technology of reconstituted tobacco, it has developed an offline testing method of hot water soluble of reconstituted tobacco with coffee machine, and the method is followed by China Tobacco Schweitzer (Yunnan) Reconstituted Tobacco Co., Ltd. in China. Hot water soluble refers to the water-soluble substance in reconstituted tobacco, which mainly come from the coating solution and are greatly related to coating rate. Up to now, there is no systematic study on the relationship between coating rate and hot water soluble at home and abroad, and no report on off-line testing method of water soluble substance to reflect the coating rate of finished products. Hot water soluble of paper-making reconstituted tobacco was tested by soxhlet extraction and coffee machine washing, the corresponding relationship between coating rate and hot water soluble testing data was compared and analyzed, so as to provide some references for the research and application of offline testing method of coating rate of papermaking reconstituted tobacco.

\section{Materials and Methods}

\subsection{Test Materials}

The base sheet and coating solution of class A reconstituted tobacco were sampled online by China Tobacco Schweitzer (Yunnan) reconstituted tobacco Co., Ltd.

Major equipment: SXT - 06 Soxhlet extractor, (Shanghai Ben-ang scientific instrument Co., Ltd), GLY - A2 Coffee machine (Beijing Gaoleya coffee machine Co., Ltd.), DHG 90706 Electro-thermostatic blast oven (Shanghai Jinghong laboratory equipment Co., Ltd.), HWS constant temperature and humidity chamber (Shanghai Jing-hong laboratory equipment Co., Ltd.), FW - 200 high-speed grinder (Beijing Zhongxing Weiye instrument Co., Ltd), BSA224S - the CW electronic balance (Sartorius scientific instrument Co., Ltd.), ZQ - 15A paper cuter (Changchun paper test machine factory).

\subsection{Test Methods}

\subsubsection{Sampling of Base Sheet and Pretreatment}

Base sheet was cut into size of $(15 \pm 1) \mathrm{cm} \times(8 \pm 1) \mathrm{cm}$, and balanced for $48 \mathrm{~h}$ under the temperature $(22+1)^{\circ} \mathrm{C}$, humidity $(60+2) \%$ in constant temperature and humidity chamber, then kept in sealed bags for later use.

\subsubsection{Testing of Moisture}

Testing of moisture was conducted according to industry standards YC/T $31<$ Tobacco and tobacco products Preparation of samples and determination of moisture Oven method $>$.

\subsubsection{Testing of Solid Content of Coating Solution}

According to national standards GB/T 1725-79 and GB/T $1725-1995,0.800 \mathrm{~g}$ coating solution is sampled and put in weighing disk, and dried under $(100+1)^{\circ} \mathrm{C}$, then cooled to room temperature (about $20 \mathrm{~min}$ is needed) and weighed, the solid content is calculated by the following formula:

$$
w=\left(m_{2}-m_{0}\right) /\left(m_{1}-m_{0}\right) \times 100
$$

Here, $w$ refers to the solid content of coating solution (\%), $\mathrm{m}_{0}$ refers to the weight of weighing disk $(\mathrm{g}), \mathrm{m}_{1}$ refers to the total weight of weighing disk and coating solution before drying $(\mathrm{g}), \mathrm{m}_{2}$ refers to the total weight of weighing disk and coating solution after drying $(\mathrm{g})$.

\subsubsection{Coating Experiment}

Samples with theoretical coating rate of $38.00 \%, 40.00 \%$, $41.00 \%, 42.00 \%, 44.00 \%$ and $46.00 \%$ were prepared respectively.

Before coating, the coating fluid was balanced under $(60+$ $5)^{\circ} \mathrm{C}$, and dried in electro-thermostatic blast oven under $(118+$ $1)^{\circ} \mathrm{C}$ to the moisture of about $11.5 \%$, then the coated samples were transferred to constant temperature and humidity chamber and balanced under temperature $(22+1)^{\circ} \mathrm{C}$, humidity $(60+2) \%$ for $48 \mathrm{~h}$. The weight of base sheet was fixed during the coating process, certain amount of coating solution calculated was coated to the base sheet totally, the coating error was controlled within the range of $\pm 1.00 \%$. Parallel coating experiments were finished, one for the test of actual coating rate, the other one for the test of hot water soluble.

\subsubsection{Test of Coating Rate}

A set of samples prepared in 1.2.4 was put into the oven, and dried for $(120 \pm 5)$ min under $(105+1)^{\circ} \mathrm{C}$, the reconstituted tobacco after drying was removed and weighed, and the coating rate $\mathrm{T}$ was calculated according to the following formula:

$$
T=\left(M_{1}-M_{2}\right) / M_{1} \times 100
$$

Here, $M_{1}$ refers to the dry weight of reconstituted tobacco after coating $(\mathrm{g})$, and $\mathrm{M}_{2}$ refers to the the dry weight of base sheet before coating $(\mathrm{g})$.

\subsubsection{Test of Hot Water Soluble}

The other set of samples prepared in 1.2.4 were grinded by a grinder and sieved through a screening mesh product with diameter of $1.00 \mathrm{~mm}$ for later use.

\section{(i) Soxhlet Extraction}

Use Q/YNZY. J 07.404- 2014< Test of hot water soluble of paper-making reconstituted tobacco Soxhlet extraction method >, the specification of China tobacco industrial Co., 
Ltd as reference, the experimental condition was oil bath heating, temperature $160^{\circ} \mathrm{C}$, solid-liquid ratio is $1: 40$, extraction time is about $1.5 \mathrm{~h}$, extracted for about 36 times, the sample was removed after extraction, and transferred to the aluminum box to be dried until constant weight under $(100+$ $1)^{\circ} \mathrm{C}$.

\section{(ii) Coffee Machine Wash Method}

According the testing method of hot water soluble in Q/CTS 001-2015, < Papermaking Reconstituted Tobacco>, the specification of China Tobacco Sweitcher (Yunnan) Reconstituted Tobacco Co., Ltd., $5.000 \pm 0.005$ g reconstituted tobacco powder prepared in 1.2.4, and washed by hot water not cooler than $85^{\circ} \mathrm{C}$ through coffee machine, the amount of water was $400 \mathrm{~mL}$ each time, and washed for 3 times until the filtered water is clear, and the samples after washing was dried until constant weight under $(100 \pm 1)^{\circ} \mathrm{C}$.

\subsection{Statistical Analysis}

The test data were statistically analyzed through Minitab 17 statistical software.

\section{Results and Analysis}

\subsection{Test Accuracy}

To verify whether experimental error was in the allowed range, the accuracy of designed experiments and measured coating rate of reconstituted tobacco in 1.2.5 was compared. As it can be seen in table 1 , the actual testing value of coating rate was higher than its designed value, it might come from the actual operation error in the process of coating which was the inevitable, but the error was small, and could be controlled smaller than $1.00 \%$, which could satisfy the accuracy requirement of quantitative coating experiment.

Table 1. Comparison of designed value and measured value of coating rate (\%).

\begin{tabular}{llll}
\hline No & Designed coating rate & Measured coating rate & Error (absolute value) \\
\hline 1 & 38.00 & 38.88 & 0.88 \\
2 & 40.00 & 40.28 & 0.28 \\
3 & 41.00 & 41.14 & 0.14 \\
4 & 42.00 & 42.45 & 0.45 \\
5 & 44.00 & 44.08 & 0.08 \\
6 & 46.00 & 46.24 & 0.24 \\
\hline
\end{tabular}

\subsection{Correlation Between Coating Rate and Hot-Water Soluble Substance Yield}

\subsubsection{Correlation Between Coating Rate and the Hot-Water Soluble Substance Yield by Soxhlet Extraction}

From table 2, it can be seen that there was positive correlation between coating rate and hot-water soluble substance yield by soxhlet extraction, and coating rate were higher than hot-water soluble substance yield. Through linear fitting, the fitting equation was obtained: $y=1.387+1.007 x$ $\left(\mathrm{R}^{2}-97.5 \%\right)$ (Figure 1). It was known that the Pearson correlation is 0.987 , and the corresponding P value is $0.000<$ 0.001 after correlation analysis, which suggested that there was strong linear correlation relationship between coating rate and hot-water soluble substance yield by soxhlet extraction, and the regression model was significant at $\alpha$ level of 0.001 .

Table 2. Comparison of coating rate and hot-water soluble substance yield by soxhlet extraction.

\begin{tabular}{llll}
\hline No. & Measured coating rate (\%) & Hot-water soluble substance yield (\%) & Pearson correlation \\
\hline 1 & 38.88 & 37.47 & 0.987 \\
2 & 40.28 & 38.68 & 0.000 \\
3 & 41.14 & 39.19 & \\
4 & 42.45 & 40.35 & \\
5 & 44.08 & 43.06 & \\
6 & 46.24 & 44.18 & \\
\hline
\end{tabular}

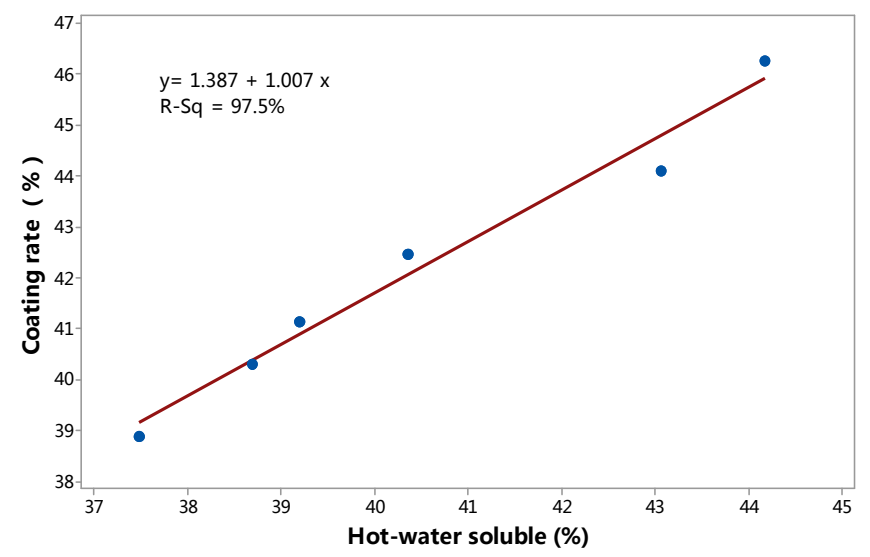

Figure 1. Correlation of coating rate and hot-water soluble substance yield by soxhlet extraction. 


\subsubsection{Correlation Between Coating Rate and the Hot-Water Soluble Substance Yield by Coffee Machine Washing}

From table 3, it can be seen that there was positive correlation between coating rate and hot-water soluble substance yield by coffee machine washing, and most measured coating rate value were a little higher than hot-water soluble substance yield. Through linear fitting, it was found that hot-water soluble substance value presented an upward trend with the increase of coating rate, the fitting equation was obtained: $y=3.184+0.932 x\left(\mathrm{R}^{2}-98.5 \%\right)$ (Figure 2). It was known that the Pearson correlation is 0.993, and the corresponding $\mathrm{P}$ value is $0.000<0.0001$ after correlation analysis, which suggested that there was strong linear correlation relationship between coating rate and hot-water soluble substance yield by coffee machine washing, and the regression model was significant at $\alpha$ level of 0.001 .

Table 3. Comparison of coating rate and hot-water soluble substance yield measured by coffee machine washing.

\begin{tabular}{llll}
\hline No. & Measured coating rate (\%) & Hot-water soluble substance yield (\%) & Pearson correlation \\
\hline 1 & 38.88 & 38.14 & 0.993 \\
2 & 40.28 & 39.57 & 0.000 \\
3 & 41.14 & 40.92 & \\
4 & 42.45 & 42.53 & \\
5 & 44.08 & 44.18 & \\
6 & 46.24 & 45.70 & \\
\hline
\end{tabular}

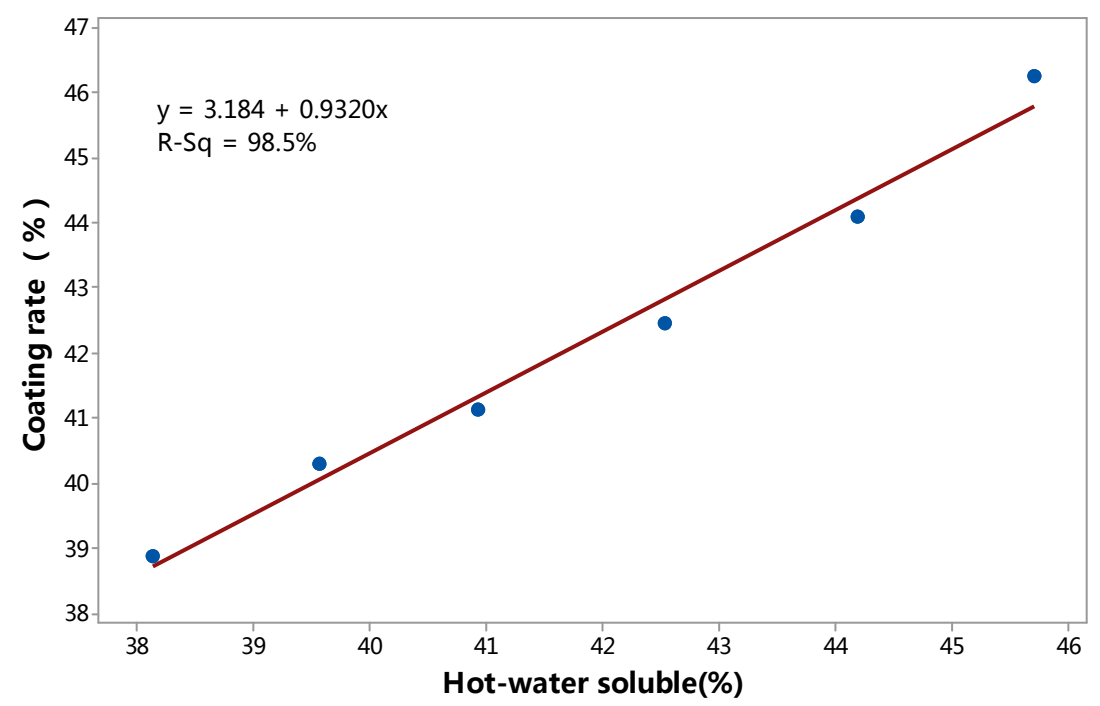

Figure 2. Correlation between coating rate and hot-water soluble substance yield measured by coffee machine washing.

\subsubsection{Comparison of Coating Rate Deduced by Hot Water Soluble Substance Yield}

Both methods of hot-water soluble substance testing were used, and according to the regression fits between coating rate and hot water soluble in 2.2.1 and 2.2.2, coating rate values were deduced, and compared with measured values, the results are shown in table 4 . The table 4 showed that the mean difference between deduced coating rate from hot-water soluble substance by soxhlet extraction and measured value was $0.67 \%$, while the mean difference between deduced coating rate and measured value by coffee machine washing was $0.36 \%$, Both differences between deduced coating rate and measured value were smaller than $1.00 \%$, so it could be concluded that the method to deduce coating rate from offline hot-water soluble substance yield was feasible.

Table 4. Comparison on measured value and deduced value of coating rate (\%).

\begin{tabular}{lllll}
\hline Item & Sampling No & $\begin{array}{l}\text { Measured yield of hot water } \\
\text { soluble substance }\end{array}$ & Deduced coating rate & Measured coating rate \\
\hline \multirow{2}{*}{ Coating rate deduced by } & 1 & 42.34 & 44.02 & 43.26 \\
soxhlet extraction & 2 & 41.87 & 43.55 & 42.94 \\
& 3 & 42.71 & 44.39 & 43.74 \\
Coating rate deduced by & 1 & 43.62 & 43.84 & 43.26 \\
coffee machine washing & 2 & 42.93 & 43.20 & 42.94 \\
\hline
\end{tabular}

Above all, there were both correlation between coating rate and hot-water soluble substance yield by soxhlet extraction and coffee machine washing, so the coating rate could be deduced by testing hot water soluble substance yield, which 
solved the problem of offline testing of coating rate. Combined with figure 1 and figure 2, the results indicated a positive linear relationship between hot-water soluble substance yield tested through both methods and coating rate, and the method of coffee machine washing was better with high determination coefficient $\mathrm{R}^{2}$ than the method of soxhlet extraction, which indicated that there was better linear correlation between hot-water soluble substance yield by the method of coffee machine washing and coating rate, and the hot water soluble substance yield through this method was closer to measured coating rate.

\section{Discussion}

Coating rate is a key control index in the produce process of paper-making reconstituted tobacco. On the one hand, its testing methods, especially the offline testing methods, are advantageous to the control of process control and ensure the stability of product quality for manufacture companies of reconstituted tobacco, on the other hand they also can help to realize the monitoring of this important index by cigarette factories.

This study combined the actual control of coating rate in reconstituted tobacco industry at present, and represented the coating rate of finished product by measuring hot-water soluble substance yield. The results showed that there was extremely significant positive linear correlation between hot water soluble substance yield and coating rate, and it was feasible to deduce coating rate by hot water soluble substance yield. This was also consistent with the research results of reference [10], but the coating rate deduced in this study was closer to the measured coating rate. Up to now, there is not uniform testing method of hot water soluble substance yield of paper-making reconstituted tobacco in China, mainly methods are extraction and washing.

Researchers in reference [12] have published a testing method of hot-water soluble substance yield by heating reflux extraction, which has the same principle with the soxhlet extraction method in the study, but the accuracy and efficiency of soxhlet extraction is higher. According to the principle of same materials having similar limit extraction yield, researchers in reference [11], have adopted washing method to extract tobacco materials on produce line, such as stem, scrap, and so on. The principle of coffee machine washing in the study is similar, and the objects to be extracted extend from tobacco raw material to paper making reconstituted tobacco products, and with the aid of coffee machine, the testing method is more convenient and accurate. In reference [11] and [12], researchers have only studied the testing methods of hot water soluble substance or modified the calculation formula, but they haven't gone into the relationship between hot water soluble and coating rate. This study has published the method of deducing coating rate from hot-water soluble substance yield by soxhlet extraction and coffee machine washing, which is simple and can be applied to practical production; For the products of same batch and same specification, what needs to be done is only to test the value of hot water soluble substance yield, and then the corresponding coating rate can be deduced, which not only shortens the testing time, but also saves manpower consumption.

Through the comparison of accuracy and applicablity of soxlet extraction and coffee machine washing, it is found that both difference between deduced and measured coating rate values are less than $1.00 \%$, while the determination coefficient $R^{2}$ of regression fit through coffee machine washing is higher than soxhlet extraction, and the coating rate deduced from the hot-water soluble substance yield with this method is closer to the measured coating rate. At the same time, the method of coffee machine washing has the advantage of easy to operate and short testing period, which makes it to be more suitable for testing in batch.

In addition, class a reconstituted tobacco products sampled online are used to analyze in this study. But for different types of reconstituted tobacco products produced by different manufacturers, the correlation between coating rate and hot water soluble substance yield is different, because the tobacco raw material, production process and product formula are all different, so the analysis and representation should be carried out based on specific testing results.

\section{Conclusion}

This study shows that there was extremely significant positive linear correlation between hot water soluble substance yield by soxhlet extration and coffee machine washing and coating rate, and the difference between deduced and measured coating rate values is less than $1.00 \%$, which indicates that the method of deducing coating rate from hot-water soluble substance yield is feasible, and the coating rate deduced from the hot-water soluble substance yield with coffee machine washing is closer to the measured coating rate. At the same time, the method of coffee machine washing has the advantage of easy to operate and short testing period, which makes it to be more suitable for practical testing.

\section{Acknowledgements}

The research is supported by Science and Technology Project of China Tobacco Yunnan Industrial Co., Ltd (2015GY02).

\section{References}

[1] Yu J J. Cigarette Technology [M]. Beijing: China Agriculture Press, 2003:322-323.

[2] Wang H Y, Xiang H Y, Liu J, Wang J, Qiu Y, Zhang Q F. Study on determination for tensile strength of paper-process reconstituted tobacco [J]. Modern Scientific Instruments, 2013 (2):133-136.

[3] Wang H W. Application Effect of Reconstituted tobacco paper in cigarette [J]. Tobacco Science, 2000(8):15-16.

[4] Wang J S, Liu X H, Ji X. Application of paper-making tobacco sheet in virginia type cigarette products $[\mathrm{J}]$. Tobacco Science, 2004 (3):9-10. 
[5] Chen Y, Jiang X D. Measurement of theoretical coating rate for paint $[\mathrm{J}]$. Coatings Technology \& Abstracts, 2004, 25(1): 38-39.

[6] Zhou W P, Chen G. Discussion methods for determination of coating rate for paint $[\mathrm{J}]$. Coatings Technology \& Abstracts, 2001, 25 (2):31-32.

[7] Tang J B, Zhao C S, Han W J. Technological improvement of paper-making process reconstituted tobacco [J]. Tobacco Science \&Technology, 2009(7):15-17.

[8] Hua Y K, Hei H, Chen J, et al. A method for improving the sensory quality of reconstituted tobacco [P]. Chinese Patent, 2012. CN102396772A.

[9] Li H, Wei Q, Liu W J, Sun Y, Zhang S D. A method to calculate rate of reconstituted tobacco by messuring the chloride content $[\mathrm{J}]$. Journal of Yunnan Agricultural University, 2014, 29 (S1): 10-14.

[10] Qiu Y, Yang X Z, Xiang H Y, et al. Study on determination method for spreading rate of paper-process reconstituted tobacco [J]. Modern Scientific Instruments, 2012(6):129-133.

[11] Dai L J, Shi J Z, Chen T. Calculation method of hot water soluble extraction rate in paper-making reconstituted tobacco leaf production line [J]. Fujian Analysis \& Testing, 2014, 23 (1): $36-38$.

[12] Hongta Tobacco (Group) Co., Ltd. A method of determination of water solubles for paper reconstituted tobacco: China, $201210447325 \quad$ [P]. 2013-04-17.http://www.pss-system. gov.cn/sipopublicsearch/search/search/showViewList.shtml

[13] Wang WS, Wang Y, YangLJ, et al. Studies on thermal behavior of reconstituted tobacco sheet [J]. Thermochimica Acta, 2005(437):7-11.

[14] Huang XM, Hu WJ, Wang YH. Research on the stabilizing Coating Rate of the Reconstituted Tobacco by Papermaking Process [J]. Paper and Paper Making, 2015, 34 (12):53-57.

[15] Zhang DK, Yan XL, Li KJ, et al. The establishment of three detection methods for coverage rate of reconstituted tobacco [J]. China Pulp\& Paper Industry, 2016, 37 (22):49-52.

[16] Fujian China Tobacco Industry Co., Ltd. A method and device for testing the coating rate of papermaking reconstituted tobacco: China, CN105675526A. 2016-06-15. http://www.pss-system.gov.cn/sipopublicsearch/patentsearch/s howSearchResult-drillSearchByViewSetting.shtml 